\title{
Genetic Algorithm Performance with Different Selection Methods in Solving Multi-Objective Network Design Problem
}

\author{
R. O. Oladele \\ Department of Computer Science \\ University of llorin \\ P.M.B. 1515, Ilorin, NIGERIA
}

\author{
J. S. Sadiku \\ Department of Computer Science \\ University of llorin \\ P.M.B. 1515, Ilorin, NIGERIA
}

\begin{abstract}
Selection is one of the key operations of genetic algorithm (GA). This paper presents a comparative analysis of GA performance in solving multi-objective network design problem (MONDP) using different parent selection methods. Three problem instances were tested and results show that on the average tournament selection is the most effective and most efficient for 10-node network design problem, while Ranking \& Scaling is the least effective and least efficient. For 21-node and 36-node network problems, Roulette Wheel is the least effective but most efficient while Ranking \& Scaling equals and outperformed tournament in effectiveness and efficiency respectively.
\end{abstract}

\section{General Terms}

Evolutionary Algorithms, Combinatorial Optimization, Artificial Intelligence.

\section{Keywords}

Genetic Algorithm, Selection Methods, Network Design Problem, Performance.

\section{INTRODUCTION}

The selection of individuals for the production of the next generation is a critical process in GA. The individuals that are chosen for reproduction and the number of children each selected individual produces are determined by the selection mechanism. The underlying principle of selection strategy is "the fitter an individual is the higher is its chance of being parent". A critical parameter to be determined in GA is the selection pressure which is the process of selecting the fittest individuals for reproduction. If it is set too low, then the rate of convergence towards the optimum solution will be too slow. If the selection pressure is set too high, the algorithm is likely to be stuck in a local optimum due to the lack of diversity in the population. Therefore, the selection methods tune the selection pressure, which in turn determines the convergence rate of the algorithm. The selection mechanism should be chosen such that convergence to the global optimum solution is guaranteed. In addition the selection mechanism should encompass knowledge of the existing data.

Different selection schemes will undoubtedly influence the performance of GA differently. This study aims at exploring the performance of GA when using different selection strategies particularly in solving Multi-Objective Network Design Problem (MONDP). MONDP is a typical example of NP-hard optimization problem
The remainder of this paper is organized as follows: Section 2 presents a brief review of literature on selection method. Selection 3 presents a brief description of MONDP while section 4 gives an overview of the genetic algorithm for MONDP. Section 5 describes in detail the selection methods that are used in the experiments. Section 6 tests the performance of GA and discusses the experimental results. The Paper is concluded in section 7.

\section{LITERATURE REVIEW ON SELECTION METHOD}

Several researchers have studied the performance of GA using differing selection schemes. However, virtually no of these researchers tested the algorithm on network design problem. The performance of GA is usually evaluated in terms of convergence rate and the number of generations to reach optimal solution [1]. Goldberg and Deb [2] were the first to study the performance of different selection schemes. They investigated the performance of proportional ranking, tournament and Genitor (steady state) selection schemes using solutions to differential equations as basis. Their investigation aimed at understanding the expected fitness ratio and convergence time. It was found that ranking and tournament selections outperformed proportional selection in terms of maintaining steady pressure towards convergence. They also showed that linear ranking and stochastic binary tournament selections have similar expectations, but recommended binary tournament selection due to its better time complexity. Goh et al. [3] focused on the selection process of GA and study common problems and solution methods of such selection schemes. They further proposed a new selection scheme called sexual selection and compared its performance with commonly used selection methods in solving some scheduling problems. It is claimed that the proposed scheme performed either on-par or better than roulette wheel selection on the average without the use of fitness scaling. In the more difficult test cases when no fitness scaling is used, the new scheme also performed better on the average when compared to tournament selection. Julstrom [4] studied the computing time efficiency of two types of rank-based selection probabilities namely linear ranking and exponential ranking probabilities in comparison with tournament selection. $\mathrm{He}$ noted that tournament selection is superior to rank-based selection because repeated tournament selection is faster than sorting the population to assign rank-based probabilities.

Mashohor et al [5] analyzed the performance of PCB inspection system with the use of three GA selection methods namely tournament, deterministic and roulette wheel selections. Their findings show that deterministic method 
requires the least computation to attain maximum fitness compared to other two methods. Zhong et al [6] did a comparative study of proportional roulette wheel selection and tournament selection, with tournament size of 6 for seven general test functions and observed that algorithm with tournament selection converges faster than the algorithm with proportional roulette wheel. Jadaan et al [7] compared proportional roulette wheel selection and rank-based selection using many mathematical fitness functions and discovered that rank-based is superior to proportional in number of generations to get optimal solution. He further observed that rank-based is steadier, faster, and more robust towards optimal

\section{MONDP}

The problem of network design consists of designing the links layout among the nodes, given a set of nodes and the mean/peak internodes traffic. The selected network (links layout) should be such that (i) certain objectives are simultaneously optimized (ii) particular constraints (requirements) are satisfied. Obviously this problem can be expressed as a multi-objective optimization problem and the problem can be as complex as the designer states it since the designer can choose as many objectives as he wants, he can also have as many kinds of links as technology and budget allow him.

In this paper, the problem is stated as an optimization of two objectives i. e., total network cost and average delay on links subject to satisfaction of flow and reliability constraints. Then, the mathematical expression of the problem can be stated as:

$$
\text { Optimize } \quad y=f(x)=\left(f_{1}(x), f_{2}(x)\right.
$$

(1)

$$
\text { Subject to: } \quad L F L O W_{i j} \leq C A P_{i j}
$$

(3)

$$
R(x) \geq R_{0}
$$

Where:

$x=\left(x_{1}, x_{2}, \ldots \ldots \ldots, x_{n}\right) \in X$ is the decision vector

$y=\left(f_{1}, f_{2}\right) \in F$ is the objective vector

$f_{1}(x)$ is the cost function of the configuration $x$

$f_{2}(x)$ is the average delay on all the links in the configuration $x$

$L F L O W_{i j}$ refers to the traffic flowing along link $\left(i_{i} j\right)$

$C A P_{i j}$ is the capacity of link $(i, j)$

$R(x)$ is the reliability of the configuration $x$

$R_{0}$ is the minimum acceptable reliability $\left(\boldsymbol{R}_{0}=0.95\right)$

The reliability calculation is done via Monte Carlo simulations.

Other network design parameters used are the followings:

$\mathrm{N}$ denotes the total number of nodes in the network

$\boldsymbol{D}_{i j}$ denotes the physical distance between every pair of nodes $\overline{\boldsymbol{i}}$ and $\boldsymbol{j}$

$\boldsymbol{C}_{i \boldsymbol{i} j}$ represents the cost of the link between nodes $\boldsymbol{i}$ and $\boldsymbol{j}$

$\boldsymbol{C}_{\tilde{i}}$ is the cost of network equipment at node $\boldsymbol{i}$ solutions than proportional roulette wheel. Razali and Geraghty [1] studied the performance of GA in solving traveling salesman problem (TSP) using different selection strategies. The results of their tests on some TSP instances reveal that tournament selection is superior to proportional roulette wheel and rank-based roulette wheel selections, obtaining best solution quality with low computing times. Results also show that tournament and proportional roulette wheel can outperform rank-based roulette wheel selection for smaller problems only and become susceptible to premature convergence as problem size increases.

$\boldsymbol{P}_{i j}$ is selection status of link $\left(\boldsymbol{i}_{j} \boldsymbol{j}\right): \boldsymbol{P}_{i j}=1$ if link $\left(\boldsymbol{i}_{j} \boldsymbol{j}\right)$ is selected, else $\boldsymbol{P}_{i j}=0$

$\mathrm{L} \quad=$ maximum distance for which the signal is sustained without amplification

$\mathrm{A}=$ cost of each amplifier unit

Poisson process was used to model the traffic delay

The objective functions; network cost and average delay are approximated by the following formulation[8]

1. Network Cost:

NetCost $=$ NodeCost + LinkCost + AmpCost

Where;

NodeCost $=\sum_{i} C_{i}$

(5)

LinkCost $=\sum_{i} \Sigma_{j} C_{i j}$

(6)

AmpCost $=\frac{\sum_{i} \Sigma_{j} D_{i f} X A}{L}$

2. Average Delay:

$A v D e l a y=\frac{\sum_{i} \Sigma_{j}\left[D E L A Y_{i j} X \quad L F L O W_{i j}\right]}{\sum_{i} \Sigma_{i} L F L O W_{i j}}$

(8)

$D E L A Y_{i j}=\frac{1}{\left[C_{i j}-\text { LFLOW }_{i j}\right]}$

$\boldsymbol{D E} \boldsymbol{L} \boldsymbol{A} \boldsymbol{Y}_{i j}=0$ if there is no link between nodes $\boldsymbol{i}$ and $\boldsymbol{j}$

$\boldsymbol{D E} \boldsymbol{L} \boldsymbol{A} \boldsymbol{Y}_{i j}=\infty$ if the network cannot handle the traffic load with the existing links' capacities and routing policy.

Breadth First Search (BFS) is used for routing and the following assumptions were made in the problem formulation The location of each network node is given

Each $\boldsymbol{C}_{i j}$ is fixed and known 
Each link is bidirectional i.e. a path can be traversed in either direction

There is no redundant link in the network

\section{GENETIC ALGORITHM FOR MONDP}

1 Initialization: randomly generate population of $\mathrm{N}$ chromosomes

2 Fitness: calculate the fitness of all chromosomes

3 Create a new population:

a. Selection: select 2 chromosomes from the population

b. Crossover: produce 2 offsprings from the 2 selected chromosomes

c. Mutation: perform mutation on each offspring.

4 Replace: replace the current population with the new population

5 Evaluation: evaluate the objective function

6 Termination: Test if the termination condition is satisfied. If so stop. If not, go to step 2 .

\section{SELECTION METHODS FOR REPRODUCTION}

\subsection{Roulette Wheel selection}

In the Roulette wheel selection method [9], the first step is to calculate the cumulative fitness of the whole population through the sum of the fitness of all individuals. After that, the probability of selection is calculated for each individual as being $P_{\text {sel }}$ pseli $=$ fi/Pfi. Then, an array is built containing cumulative probabilities of the individuals. So, $\mathrm{n}$ random numbers are generated in the range 0 to Pfi and for each random number an array element which can have higher value is searched for. Therefore, individuals are selected according to their probabilities of selection

\subsection{Tournament Selection}

Tournament selection is probably the most popular selection method in genetic algorithm due to its efficiency and simple implementation [2]. In tournament selection, $n$ individuals are selected randomly from the larger population, and the selected individuals compete against each other. The individual with the highest fitness wins and will be included as one of the next generation population. The number of individuals competing in each tournament is referred to as tournament size, commonly set to 2 (also called binary tournament). Tournament selection also gives a chance to all individuals to be selected and thus it preserves diversity, although keeping diversity may degrade the convergence speed. The tournament selection has several advantages which include efficient time complexity, especially if implemented in parallel, low susceptibility to takeover by dominant individuals, and no requirement for fitness scaling or sorting $[2,10]$.

In tournament selection, larger values of tournament size lead to higher expected loss of diversity $[10,11]$. The larger tournament size means that a smaller portion of the population actually contributes to genetic diversity, making the search increasingly greedy in nature. There might be two factors that lead to the loss of diversity in regular tournament selection; some individuals might not get sampled to participate in a tournament at all while other individuals might not be selected for the intermediate population because they lost a tournament

\subsection{Ranking and Scaling Selection}

The scaling method maps raw objective function values to positive real values, and the survival probability for each chromosome is determined according to these values. Fitness scaling has a twofold intention: to maintain a reasonable differential between relative fitness ratings of chromosomes; and to prevent too-rapid takeover by some dominant chromosomes to meet the requirement to limit competition early but to stimulate it later.

\section{COMPUTATIONAL EXPERIMENTS AND RESULTS}

Table 1:Results for 10-node network problem

\begin{tabular}{|c|c|c|c|c|c|c|c|c|c|c|c|c|}
\hline \multirow{2}{*}{$\begin{array}{c}\text { No of } \\
\text { Gen }\end{array}$} & \multicolumn{4}{|c|}{ ROULETTE WHEEL } & \multicolumn{3}{|c|}{ RANKING AND SCALING } & \multicolumn{3}{c|}{ TOURNAMENT } \\
\cline { 2 - 14 } & C/Ratio & Cost & Delay & $\begin{array}{c}\text { Comp. } \\
\text { Time }\end{array}$ & C/Ratio & Cost & Delay & $\begin{array}{c}\text { Comp. } \\
\text { Time }\end{array}$ & C/Ratio & Cost & Delay & Comp. Time \\
\hline 5 & 100 & 534.0 & 0.07 & 134 & 100 & 637.6 & 0.08 & 152 & $\mathbf{1 0 0}$ & $\mathbf{5 3 4 . 0}$ & $\mathbf{0 . 0 4}$ & $\mathbf{1 5 4}$ \\
\hline $\mathbf{1 0}$ & $\mathbf{1 0 0}$ & $\mathbf{5 3 4 . 0}$ & $\mathbf{0 . 0 6}$ & $\mathbf{3 4 4}$ & $\mathbf{1 0 0}$ & $\mathbf{6 3 7 . 6}$ & $\mathbf{0 . 0 7}$ & $\mathbf{3 9 0}$ & 100 & 655.4 & 0.08 & 361 \\
\hline 15 & 100 & 534.0 & 0.06 & 450 & 100 & 637.6 & 0.07 & 498 & 100 & 534.0 & 0.08 & 552 \\
\hline 20 & 100 & 534.0 & 0.06 & 570 & 100 & 637.6 & 0.07 & 504 & 100 & 534.0 & 0.08 & 603 \\
\hline
\end{tabular}


Table 2: Results for 21-node network problem

\begin{tabular}{|c|c|c|c|c|c|c|c|c|c|c|c|c|}
\hline \multirow{2}{*}{$\begin{array}{l}\text { No of } \\
\text { Gen }\end{array}$} & \multicolumn{4}{|c|}{ ROULETTE WHEEL } & \multicolumn{4}{|c|}{ RANKING AND SCALING } & \multicolumn{4}{|c|}{ TOURNAMENT } \\
\hline & C/Ratio & Cost & Delay & $\begin{array}{l}\text { Comp. } \\
\text { Time }\end{array}$ & C/Ratio & Cost & Delay & $\begin{array}{l}\text { Comp. } \\
\text { Time }\end{array}$ & C/Ratio & Cost & Delay & $\begin{array}{c}\text { Comp. } \\
\text { Time }\end{array}$ \\
\hline 5 & 98 & 1305 & 0.07 & 837 & 95 & 1166.8 & 0.06 & 937 & 100 & 1265.8 & 0.06 & 998 \\
\hline 10 & 100 & 1167.4 & 0.06 & 1482 & 98 & 1166.8 & 0.05 & 1499 & 100 & 1265.8 & 0.06 & 1546 \\
\hline 15 & 100 & 1265.8 & 0.05 & 4143 & 100 & 1163.0 & 0.07 & 4743 & 100 & 1265.8 & 0.05 & 4943 \\
\hline 20 & 100 & 1265.8 & 0.06 & 5434 & 100 & 1163.0 & 0.05 & 6312 & 100 & 1163.0 & 0.05 & 7854 \\
\hline
\end{tabular}

Table 3: Results for 36-node network problem

\begin{tabular}{|c|c|c|c|c|c|c|c|c|c|c|c|c|}
\hline \multirow{2}{*}{$\begin{array}{c}\text { No of } \\
\text { Gen }\end{array}$} & \multicolumn{3}{|c|}{ ROULETTE WHEEL } & \multicolumn{3}{|c|}{ RANKING AND SCALING } & \multicolumn{4}{c|}{ TOURNAMENT } \\
\cline { 2 - 15 } & C/Ratio & Cost & Delay & $\begin{array}{c}\text { Comp. } \\
\text { Time }\end{array}$ & C/Ratio & Cost & Delay & $\begin{array}{c}\text { Comp. } \\
\text { Time }\end{array}$ & C/Ratio & Cost & Delay & $\begin{array}{c}\text { Comp. } \\
\text { Time }\end{array}$ \\
\hline 5 & $\mathbf{9 8}$ & $\mathbf{1 3 0 5}$ & $\mathbf{0 . 0 5}$ & $\mathbf{2 3 3 3}$ & 98 & 1305 & 0.07 & 2454 & 98 & 1305 & 0.07 & 2454 \\
\hline 10 & 100 & 1259 & 0.07 & 3771 & 100 & 1167.4 & 0.06 & 3672 & 100 & 1167.4 & 0.06 & 3672 \\
\hline 15 & 100 & 1259 & 0.08 & 7043 & 100 & 1259 & 0.06 & 7112 & 100 & 1259 & 0.06 & 7112 \\
\hline 20 & 100 & 1167.4 & 0.06 & 12980 & $\mathbf{9 8}$ & $\mathbf{1 1 6 7 . 4}$ & $\mathbf{0 . 0 5}$ & $\mathbf{9 7 1 6}$ & $\mathbf{9 8}$ & $\mathbf{1 1 6 7 . 4}$ & $\mathbf{0 . 0 5}$ & $\mathbf{9 7 1 6}$ \\
\hline 25 & 100 & 1259 & 0.09 & 13090 & 100 & 1167.4 & 0.05 & 12273 & 100 & 1167.4 & 0.05 & 19273 \\
\hline
\end{tabular}

\section{CONCLUSION}

In this paper three types of selection strategy in the GA procedure to solve network topology design problem are described. Their relative performances in terms of solution quality and computation time are compared. From the results of experiment on three randomly generated networks. On the average, tournament selection returns the best solution both in quality and computation time while the solution returned by Ranking \& Scaling is the worst both in quality and computation time for 10-node network. For 21-node and 36node networks, Ranking \& Scaling performed as well as tournament selection in solution quality but outperformed tournament in computation time. Roulette wheel has the worst solution quality but the best computation time

\section{ACKNOWLEDGEMENT}

The authors acknowledge Hammed Sanusi who has offered programming assistance.

\section{REFERENCES}

[1] Razali, N. M., Geraghty, J. 2011. Genetic Algorithm Performance with Different Selection Strategies in Solving TSP. In Proceedings of the World Congress on Engineering, vol. II, London, UK.

[2] Goldberg, D. E. and Deb Kalyanmoy. 1991. A Comparative Analysis of Selection Schemes Used in
Genetic Algorithms. In: G.J.E. Rawlins (Ed), Foundations of Genetic Algorithms, Morgan Kaufmann, Los Altos, 69 - 93 .

[3] Goh, K. S., Lim, A., Rodrigues, B. 2..3. Sexual Selection for Genetic Algorithms. Artificial Intelligence Review 19: 123 - 152, Kluwer Academic Publishers.

[4] Julstrom, B. A. 1999. It's All the Same to Me: Revisiting Rank-Based Probabilities and Tournaments, Department of Computer Science, St. Cloud State University.

[5] Mashohor, S.Evans, J. R., Arslan, T. 2005. Elitist Selection Schemes for Genetic Algorithm based Printed Circuit Board Inspection System, Department of Electronics and Electrical Engineering, University of Edinburgh, 974 - 978.

[6] Zhong, J., Hu, X., Gu, M., Zhang, J. 2005. Comparison of Performance between Different Selection Strategies on Simple Genetic Algorithms. In Proceeding of the International Conference on Computational Intelligence for Modelling, Control and automation, and International Conference of Intelligent Agents, Web Technologies and Internet Commerce.

[7] Jadaan, O.A., Rajamani, L., Rao, C. R. 2005. Improved Selection Operator for GA. Journal of Theoretical and Applied Information Technology. 
[8] Banerjee, N., Kumar, R. 2007. Multiobjective Network Design for Realistic Traffic Models. In Proc. Genetic and Evolutionary Computations Conference (GECCO07), 1904-1911.

[9] Holland, J. H. 1992. Adaptation in Natural and Artificial Systems, 2nd Ed, MIT Press.

[10] Blickle, T, Thiele, L. A. 1995. Comparison of Selection Schemes used in Genetic Algorithms. TIK-Report, Zurich.
[11] Whitley, D. 1989. The genitor algorithm and selection pressure: Why rank-based allocation of reproductive trials is the best. In Proceeding of the 3rd International Conference on Genetic Algorithms.

[12] Sivaraj, R.,Ravichandran, T. 2011. A Review of Selection Methods in Genetic Algorithm. International Journal of Engineering Science and Technology (IJEST). Vol.3, no. 5, $3792-3797$. 